\title{
Epigenetic Inheritance and Reprogramming in Plants and Fission Yeast
}

\author{
R.A. Martienssen, A. Kloc, R.K. Slotkin, and M. Tanurdží́ \\ Cold Spring Harbor Laboratory, Cold Spring Harbor, New York 11724
}

\begin{abstract}
Plants and fission yeast exhibit a wealth of epigenetic phenomena, including transposon regulation, heterochromatic silencing, and gene imprinting. They provide excellent model organisms to address the question of how epigenetic information is propagated to daughter cells. We have addressed the questions of establishment, maintenance, and inheritance of heterochromatic silencing using the fission yeast Schizosaccharomyces pombe and the plant Arabidopsis thaliana by using a variety of genetic and genomic approaches. We present here results showing the cell cycle dependence of RNA in fission yeast RNA interference (RNAi), which is required for proper transcriptional silencing of the centromeric heterochromatin, and that this process occurs during $\mathrm{S}$ phase, allowing for precise copying and reestablishment of heterochromatic histone modifications following DNA replication and cell division. We also show that in plants, cells in culture and male germ-line cells undergo massive epigenomic changes correlated with the appearance of a novel class of 21-nucleotide small interfering RNA (siRNA) from transcriptionally reactivated transposable elements (TEs) following loss of heterochromatic DNA and histone methylation. We propose a model for the role of deliberate TE reactivation in germ-line companion cells as part of a developmental mechanism for first revealing and then silencing TEs via small RNA, which may contribute to reprogramming during early development in plants and animals.
\end{abstract}

Eukaryotic genomes are traditionally characterized as euchromatic or heterochromatic, originally based on their staining patterns (Heitz 1928). These patterns correspond to domains of different chromatin compaction levels, with euchromatin being the less compact of the two. Heterochromatin formation and maintenance have important roles in genome stability and centromere function as well as transcriptional silencing of various TEs and position effect variegation in eukaryotes, $\mathrm{X}$ chromosome inactivation in mammals, and gene imprinting in plants and animals (Lippman and Martienssen 2004; Henderson and Jacobsen 2007; Zaratiegui et al. 2007). The two chromatin states also differ in their content and transcriptional activity: Euchromatin is largely composed of genes, whereas heterochromatin is composed of various repeats, including all classes of TEs (Lippman and Martienssen 2004). The difference in composition of the two compartments is reflected in their transcriptional activity: Euchromatin is traditionally viewed as the active portion of the genome, transcribed in response to regulatory cues, whereas heterochromatin is considered transcriptionally silenced, although, paradoxically, it must be transcribed in order to be silenced (Volpe et al. 2002).

The molecular and cytological distinctions of the two chromatin compartments are established by a combination of DNA and histone modifications (Martienssen and Colot 2001; Henderson and Jacobsen 2007). In both plants and fission yeast, heterochromatic DNA is associated with a histone octamer carrying a dimethylation mark at lysine 9 on the amino terminus of histone H3 (H3K9me2). In plants, and Arabidopsis in particular, heterochromatic DNA is also heavily methylated, with 5-methyl cytosine $(5 \mathrm{mC})$ found in all three possible sequence contexts found in plants: $\mathrm{CG}, \mathrm{CHG}$, and $\mathrm{CHH}$ (Henderson and Jacobsen 2007). The targeting of heterochromatic DNA and histone modifications is achieved through the action of RNAi, which provides the sequence specificity necessary to guide the silencing complexes containing DNA and histone modifiers (Zaratiegui et al. 2007).

\section{EPIGENETIC INHERITANCE IN FISSION YEAST}

The three centromeres of the fission yeast $S$. pombe have been an excellent system to study the molecular mechanisms that establish heterochromatic silencing and its inheritance upon cell division. Their repetitive structure is very similar, but simplified, compared to higher eukaryotic centromeres (Smith and Corces 1995). The central centromeric region is the site of kinetochore binding that is flanked by innermost repeats which, in turn, are flanked by the outermost repeats consisting of $d g$ and $d h$ repeat sequences. The $d g$ and $d h$ repeats are enriched in H3K9me2 (Volpe et al. 2003), which is bound by the fission yeast homolog of the heterochromatic protein 1 (HP1) Swi6 (Holm et al. 2005). This, in turn, is necessary for proper attachments of the kinetochore (Nonaka et al. 2002). Targeting of the heterochromatic chromatin modifications is accomplished by RNAi (Volpe et al. 2002).

RNAi mechanisms take advantage of the $d h$ and $d g$ transcripts being generated from both DNA strands by RNA polymerase II (PolII) (Volpe et al. 2002). The sole fission yeast Argonaute protein Agol cotranscriptionally slices the nascent heterochromatic transcripts (Irvine et al. 2006). This is hypothesized to create sites for the RNA-dependent RNA polymerase (RDRC) complex, which results in formation of double-stranded RNA molecules. These are quickly processed into 21-24-nucleotide siRNA molecules by the Dicer protein Dcr1 (Colmenares et al. 2007). The siRNA molecules then serve as guides when they are bound 
by Ago1 and incorporated into the RNA-induced transcriptional silencing (RITS) complex (Verdel et al. 2004). This is required for the final step of heterochromatin formation, whereby the RITS complex interacts, through an unknown mechanism, with the Rik1 complex that contains the histone methyltransferase $\mathrm{Cl} 4$ responsible for $\mathrm{H} 3 \mathrm{~K} 9 \mathrm{me} 2$ methylation (Horn et al. 2005). Ultimately, H3K9me2 is recognized by the chromodomain-containing Swi6, which itself is bound by cohesins (Holm et al. 2005), ensuring the proper functioning of fission yeast centromeres and chromosomal segregation during cell division.

\section{Heterochromatin and the Cell Cycle in Fission Yeast}

Given the basic premises of chromatin replication, it is clear that the need for the RNAi-targeted heterochromatin formation process differs between the stages of the cell cycle. It also poses a problem for heterochromatin inheritance, following cell division, because, in the case of fission yeast, the epigenetic information is carried by the histone marks that themselves are not inherited (Kloc and Martienssen 2008).

We have addressed the question of heterochromatin inheritance in fission yeast by profiling $d g$ and $d h$ transcripts, siRNAs, and histone modifications throughout the cell cycle. Contrary to most eukaryotes, fission yeast heterochromatin is known to replicate during early $\mathrm{S}$ phase (Kim and Huberman 2001). This is precisely the time in the cell cycle when the $d g$ and $d h$ centromeric transcripts can first be detected in cells arrested in early S phase (Kloc et al. 2008), concomitant with the localization of PolII in the same regions (Chen et al. 2008). The $d g$ transcripts often appear slightly ahead of the $d h$ transcripts, which may be due to the two repeats being located at different distances from the origins of replication. At the same time, H3K9me 2 local concentration is at its lowest at the same time in the cell cycle. With the progression of heterochromatic DNA replication past early $\mathrm{S}$ phase, an increase in the RNAi factors can be seen, as well as the appearance of heterochromatic siRNA. This indicates that RNAi occurs specifically in the S phase (Kloc et al. 2008). Consequently, this is followed by an increase in $\mathrm{H} 3 \mathrm{~K} 9 \mathrm{me} 2$, upon the finish of DNA replication, and the faithful reestablishment of heterochromatin. The H3K9me2 levels further increase, peaking during the $\mathrm{G}_{2}$ phase of the cell cycle.

The loss of Swi6 binding in heterochromatic repeats is the earliest event detected so far that leads to cell-cyclespecific phasing in heterochromatin transcriptional activity, RNAi activity, and histone modification. Swi6 binding depends on the presence of $\mathrm{H} 3 \mathrm{~K} 9 \mathrm{me} 2$; however, Swi6-H3K9me2 interaction is further dependent on the so-called phosphomethyl switch (Fischle et al. 2003). This term refers to the presence or absence of a phosphate group on histone H3 serine10 (H3S10p), located directly next to the lysine 9 residue carrying the dimethylation modification. In the presence of H3S10p, the Swi6$\mathrm{H} 3 \mathrm{~K} 9 \mathrm{me} 2$ interaction is abolished and the transcriptional silencing of the region is lost (Chen et al. 2008; Kloc et al. 2008). Phosphorylation of H3S10 is under the control of the aurora-B kinase Ark1.
Taken together, these results point to a cell-cycle-regulated and coordinated series of molecular events that ensure that epigenetic information contained within parental chromosomes is faithfully transmitted to daughter chromosomes, even in the absence of a directly heritable epigenetic mark such as DNA methylation.

\section{EPIGENETIC INHERITANCE IN PLANTS}

Plants offer the full palette of epigenetic modifications, both at the level of DNA methylation and numerous histone modifications. This potential for epigenetic control in plants impacts plant development, responses to biotic and abiotic stress, and integration of environmental and ontogenic cues in the transition to flowering (Henderson and Jacobsen 2007). Perhaps one of the best-studied epigenetic systems in plants is heterochromatin regulation (Lippman and Martienssen 2004).

\section{Heterochromatic Changes in Proliferating Immortalized Plant Cells}

Plant cell culture is a known source of induced genetic and epigenetic variation (Hirochika 1993; Kaeppler et al. 2000). A number of plant species when grown in culture undergo transposon reactivation and a number of genes undergo epigenetic changes that lead to epiallele formation (Grandbastien et al. 1989; Kaeppler et al. 2000). This represents a good model for studying the epigenetic mechanisms involved in establishment and maintenance of heterochromatin in a higher eukaryote such as Arabidopsis.

We first examined a compendium of gene expression profiles from various organs and cell types from Arabidopsis, including a long-term cell suspension culture (Schmid et al. 2005). All of the gene expression profiles contained within this set were created using the Affymetrix ATH1 gene expression platform. This array contains about 1200 specific oligonucleotide probes matching various copies of transposable elements in the Arabidopsis genome. This analysis showed that a large number of TEs (mainly those that represent relatively intact copies in the genome) become reactivated in cell suspension culture, whereas TEs remain transcriptionally silenced in Arabidopsis leaves and callus samples.

Transcriptional reactivation of TEs in plants is accompanied by changes in epigenetic chromatin modifications. This can be accomplished by down-regulation or by mutations in key regulators of heterochromatin: DNA methyltransferases (MET1, CHROMOMETHYLTRANSFERASE3 [CMT3], and DOMAINS REARRANGED METHYLATRANSFER$A S E 1$ and 2 [DRM1 and DRM2], chromatin remodeler $D E$ CREASE IN DNA METHYLATION1 [DDMI], histone methyltransferases, in particular the major H3K9me2 methyltransferase KRYPTONITE (KYP), as well as some components of the RNAi pathway (Lippman et al. 2003). The $d d m 1$ mutation has been particularly well studied in the context of TE regulation, because most TEs in the Arabidopsis genome become reactivated in this background (Singer et al. 2001; Lippman et al. 2003). This is accompanied by a nondiscriminating loss of heterochromatic DNA and H3K9me2 methylation (Lippman et al. 2004). 
We therefore analyzed the chromatin states of immortalized Arabidopsis cells grown in a long-term suspension culture using a custom-made tiling microarray of Arabidopsis chromosome 4 to determine the DNA and histone methylation patterns (http://chromatin.cshl.edu/epiculture/). As predicted, we detected significant hypomethylation of heterochromatic DNA and loss of $\mathrm{H} 3 \mathrm{~K} 9 \mathrm{me} 2$, often replaced by the euchromatic histone modification $\mathrm{H} 3 \mathrm{~K} 4 \mathrm{me} 2$. Interestingly, these changes only affected some families of TEs, whereas other TEs, often spatially juxtaposed to the hypomethylated TEs, remained decorated with heterochromatic marks. Our experiments also revealed that these patterns are stable and mitotically heritable throughout many cell divisions. Because loss of heterochromatic marks was specific to some but not all TE families, we postulated that this was not an effect of transcriptional down-regulation of numerous chromatin regulators. Indeed, expression profiling revealed that all of the known chromatin regulators are overexpressed in cell culture, including all classes of DNA and histone methyltransferases and proteins involved in RNAi. The only protein whose expression was lost in cell culture was ROS1, a DNA demethylase (Zhu et al. 2007). We then used next-generation Illumina DNA sequencing to profile small RNA molecules in this sample and compared this profile to the small RNA profiles of young rosette leaves and freshly induced callus samples. This analysis revealed drastic shifts in siRNA abundance and length specific to those TE families that lost heterochromatic marks, with a shift from the predominant 24-nucleotide-size class to the 21-nucleotide size class whose production is under different genetic control (Fig. 1) (Brodersen and Voinnet 2006). This change was particularly striking for the demethylated and reactivated family of Athila retrotransposons (Fig 1). It therefore seems that this failure to specifically target appropriate heterochromatic marks upon cell division is due to perturbations in the RNAi systems used to target heterochromatic modifications. However, the fact that the heterochromatic state of other TE families, such as Atlantys, is faithfully reproduced over many cell divisions reflects the general availability of the core RNAi machinery and other chromatin regulators. The loss of heterochromatic modification was also detected in short regions of homology with TEs such as short interspersed repetitive elements (SINEs). This can have a particularly significant effect on gene regulation because SINEs are often found in the vicinity of genes. In the case of the imprinted gene $F W A$ (Kinoshita et al. 2004), the SINE known to be the imprinting site in planta, becomes hypomethylated in cell culture, with a 25 -fold increase in transcription at the $F W A$ locus.

Concurrently with the loss of heterochromatic silencing in TEs, we also detected genic hypermethylation in these immortalized plant cells. Although the majority of hypermethylation is located in the coding regions and corresponds to an increase in the novel gene body $\mathrm{CpG}$ methylation (Vaughn et al. 2007), a number of genes undergo epiallele formation in these cells. This was evidenced by the concomitant appearance of novel DNA and $\mathrm{H} 3 \mathrm{~K} 9 \mathrm{me} 2$ methylation. In addition, close to $90 \%$ of these new epialleles, including an epiallele at the AGAMOUS $(A G)$ locus, a gene known to be prone to epiallele formation (Jacobsen et al. 2000), also have novel 24-nucleotide
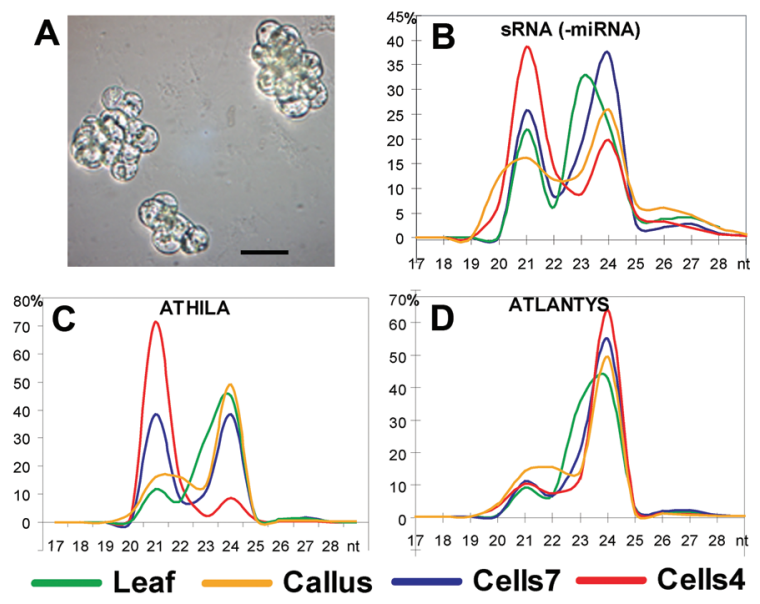

Figure 1. Size distribution of siRNA sequences in plant cell suspension culture $(A)$, wild-type (Col) leaves, and wild-type (Col) callus. The relative frequencies of heterochromatic small RNAs $(B)$ and those matching exclusively Arabidopsis retrotransposon families Athila $(C)$ and Atlantys $(D)$ were plotted according to size in cell culture 4 days and 7 days after transfer to fresh medium. The 21-nucleotide siRNAs accumulate only in cultured cells but not wild-type leaf or callus samples and correspond to Athila elements (which lose methylation), but not to Atlantys elements (which do not). (Modified from Tanurdžić et al. 2008.)

siRNA associated with them in cell culture. It is tempting to speculate that the appearance of novel epialleles in cell culture may be, at least partially, due to the inability of the cells to prune RNA-directed DNA methylation due to transcriptional down-regulation of ROS1 (Zhu et al. 2007). Intriguingly, the proximal ROS1 promoter contains a fragment of a helitron TE that becomes hypermethylated in cell culture, and thus, ROS1 may itself be prone to epiallele formation.

\section{Epigenetic Reprogramming and Plant Germ Cells}

In addition to TE reactivation in cell culture, reexamination of previous Arabidopsis expression profiling data (Schmid et al. 2005) revealed that the transcriptional reactivation of TEs also occurs in mature wild-type pollen (Slotkin et al. 2009). Unlike animals, plant meiocytes differentiate from adult somatic cells late in development. The pollen grain is produced from two subsequent mitotic divisions that occur after meiosis, so that pollen contains three haploid cells. The largest is the pollen vegetative cell, controlled by the pollen vegetative nucleus, which contains decondensed chromatin. Enclosed within the pollen vegetative cell are two sperm cells with compact chromatin. The vegetative nucleus does not contribute genetic material to the next generation, whereas the two sperm cells are involved in the double fertilization process typical of most seed plants, providing paternal genetic and epigenetic contributions to the zygote and the endosperm.

Our analysis of TE transcriptional activity of pollen using Arabidopsis microarray data (Becker et al. 2003; Honys and Twell 2004; Pina et al. 2005; Schmid et al. 
2005) showed that although TEs are generally silenced throughout the plant, they are up-regulated in pollen. This co-coordinated TE up-regulation was not dependent on the chromosomal position of TEs nor was it specific to particular families of TEs. Therefore, we concluded that TE transcriptional reactivation in pollen is due to a loss of particular trans-acting silencing factors.

Using gene-trap and enhancer-trap reporter lines inserted into TEs (Sundaresan et al. 1995), we assayed the precise location and timing of TE reactivation in the pollen grain. As expected, no GUS staining was detected in the plant body; however, strong GUS staining was detected in mature pollen for all 24 different reporter lines with insertion in various TEs throughout the Arabidopsis genome. Furthermore, upon closer inspection, we were able to confirm that the positive GUS-staining signal in pollen was in the pollen grain cytoplasm, whose content is under the control of the vegetative nucleus (VN). In contrast, no GUS accumulation was detected in the sperm cells (SCs). We further tested the hypothesis that TE reactivation is specific to the VN by looking at novel, heritable transposition events using transposon display. We could detect numerous transposition events in the pollen DNA itself, but we were not able to detect any novel transposition in $500 \mathrm{~F}_{1}$ plants. This evidence also supports the notion that pollen TE reactivation is specific to the $\mathrm{VN}$ and does not occur in the SC. In addition, microarray data and reverse transcriptase-polymerase chain reaction (RTPCR) both demonstrate that TEs are not expressed in SCs purified from the pollen grain using fluorescence-activated cell sorting (FACS) (Borges et al. 2008).

What is the extent, then, of epigenetic changes in the VN chromatin that allow transcriptional TE reactivation? We assayed DNA methylation by sequence analysis of bisulfite-converted DNA from pollen as well as purified SCs using TE-specific primers in PCR amplification. The results of this experiment showed that, on average, TEs are hypomethylated in the genomic DNA isolated from pollen, retaining some DNA methylation. In particular, methylation seems to be lost from the asymmetric methylation sites, which is targeted by siRNA. In contrast, SC DNA contains fully methylated cytosines in both symmetric and asymmetric sequence contexts. This result implicated a VN-specific down-regulation of one or more genes required for heterochromatic silencing in Arabidopsis and may be accompanied by active DNA demethylation specifically targeted to the VN. RT-PCR analysis showed that of several candidate genes known to regulate TE and heterochromatic silencing, genes involved in the production of the 24nucleotide siRNA (particularly RNA DEPENDENT RNA POLYMERASE2 [RDR2] and DICER-LIKE3 [DCL3]) are down-regulated in pollen, whereas the trans-acting siRNA and microRNA (miRNA)-generating pathways (21nucleotide trans-acting siRNA and miRNA) seemed to be fully functional. Importantly, we also showed that the master regulator of heterochromatic silencing, DDM1 protein, does not accumulate in the VN but does in the SCs.

We investigated the role of siRNA in targeting heterochromatic silencing in the male germ line by highthroughput sequencing of small RNA molecules from pollen and isolated SCs and compared them to small RNA data sets from wild-type Arabidopsis leaves and inflorescences as well as $d d m 1$ inflorescences. When the small RNA sequences are aligned to the reference genome, a striking similarity in the patterns and size of matching siRNAs is seen between the two samples with epigenetically misregulated TEs: $d d m 1$ plants and pollen (Fig. 2). Most TEs, such as AtMul, seem to lose or have significantly reduced levels of the heterochromatic 24nucleotide siRNA in both $d d m 1$ (Lippman et al. 2003) and pollen. In addition, the largest family of TEs in the Arabidopsis genome, the Athila retrotransposons, show a massive increase in the 21-nucleotide siRNA in both samples (Fig. 2). We therefore conclude that upon epigenetic reactivation of TEs, a novel class of siRNA, the 21nucleotide in size, and matching Athila TEs, is massively up-regulated.

Interestingly, the 21-nucleotide siRNA from reactivated TEs accumulate in the SC small RNA library (Fig. 2 ), even though the primary TE transcripts are not found in the SC RNA. This result would imply that the 21nucleotide siRNA can move between the VN and SC. We tested whether small RNAs could communicate from the $\mathrm{VN}$ to the SC by expressing an artificial miRNA (amiRNA) in the VN (Engel et al. 2005; Schwab et al. 2006) and targeting a green fluorescent protein (GFP)

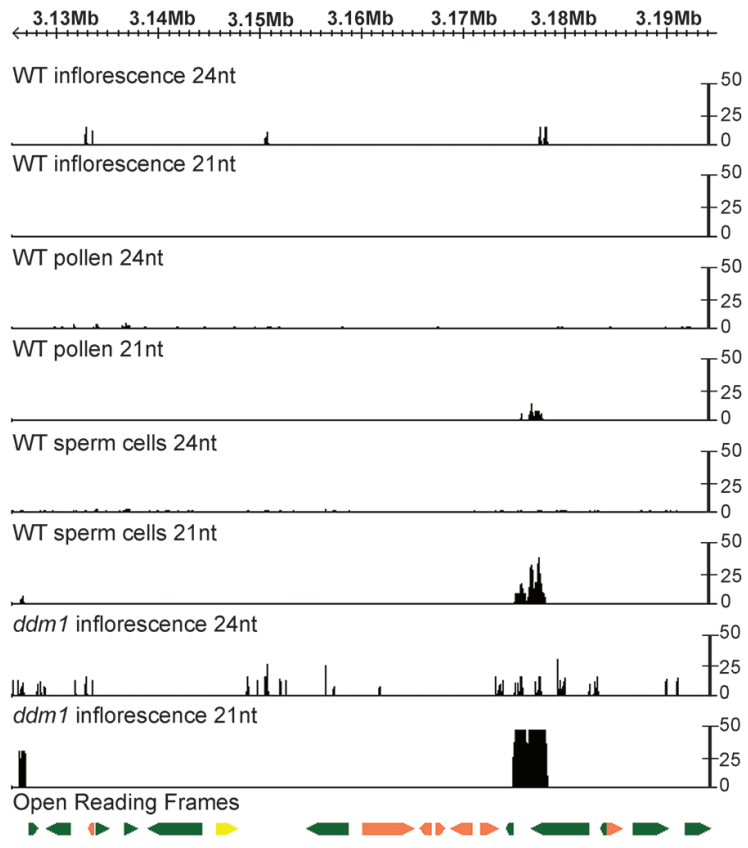

Figure 2. siRNA distribution according to size, frequency, and sequence homology in Arabidopsis pollen, sperm cells, and a $d d m 1$ mutant. Distributions of 21- and 24-nucleotide siRNAs are shown for a $65-\mathrm{kb}$ section of pericentromeric heterochromatin from chromosome 4. Gene and ORF annotations include DNA transposons (red), retrotransposons (green), and gene (yellow). Bars represent copy-corrected counts per read of 21- and 24nucleotide siRNAs in 100-bp windows for wild-type inflorescence, wild-type pollen, $d d m 1$ inflorescence, and wild-type sperm cells. In pericentromeric heterochromatin, large peaks of 21-nucleotide siRNAs in $d d m 1$, sperm cells, and pollen match Athila family retrotransposons. 


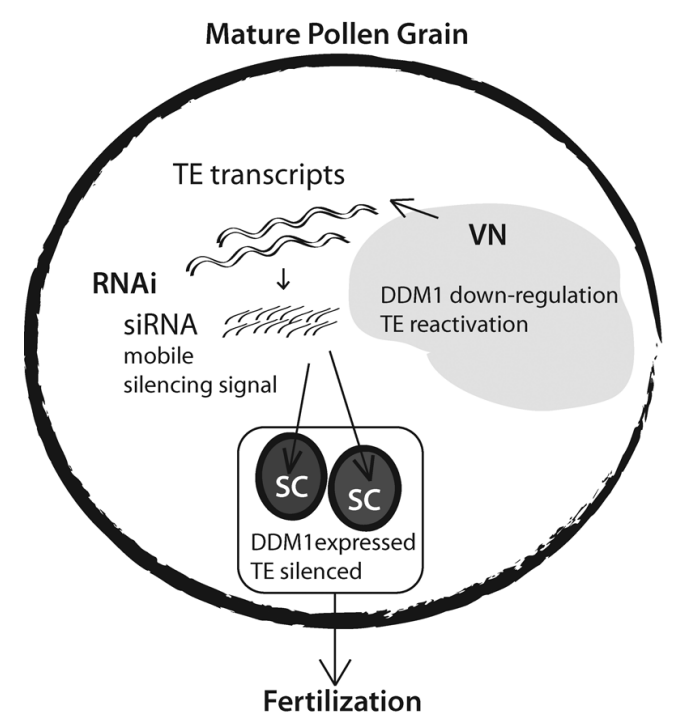

Figure 3. Model of TE reactivation and its role in the reprogramming of the germ line. The pollen $\mathrm{VN}$ and SCs differ in DDM1 localization and TE expression. Reactivated TEs, such as Athila retrotransposons, from the VN produce 21-nucleotide siRNAs via RNAi. This novel class of siRNA is mobile and accumulates in the SC. Thus, TEs are "revealed" by epigenetic reprogramming of the $\mathrm{VN}$ in order to target and silence themselves in the SC.

reporter expressed specifically in the SC (Engel et al. 2005). In the plants homozygous for both the amiRNA and GFP reporter, we could detect almost complete silencing of the GFP reporter in the SC. These data further corroborate our hypothesis that small RNA can move between the two nuclear compartments in pollen and communicate with the silencing signal generated in the $\mathrm{VN}$ to the $\mathrm{SC}$ to ensure TE silencing and both the genetic and epigenetic stability of germ cells (Fig. 3).

\section{SMALL RNA-MEDIATED TRANSPOSON CONTROL IN ANIMALS AND PROTISTS}

Our results may shed light on transposon regulation in animals as well as in plant germ cells and even in protists such as Tetrahymena and Paramecium. In plants and protists, genomic reprogramming seems to be confined to companion nuclei, such as the vegetative nucleus in pollen and the macronucleus in protists, which undergoes DNA elimination and RNAi and does not contribute to the next generation. In mammals, transposon silencing is transiently lost through extensive reprogramming, both in the germ line and in the early embryo. An ancient conserved mechanism of reprogramming may underlie transposon activation in each case.

Recently, there has been a great deal of interest in germ-line small RNA in Drosophila and the mouse. Much of this research has focused on Piwi-interacting RNA (piRNA), a 25-29-nucleotide small RNA associated with the PIWI class of Argonaute proteins (which are apparently not found in plants) (Aravin et al. 2007a; Gunawardane et al. 2007). Although their biogenesis is still somewhat obscure, it depends on the PIWI proteins themselves (Aravin et al. 2007a; Gunawardane et al. 2007). Retrotransposon targeting is achieved by sequence specificity of piRNAs that arise from genomic locations with sequences containing antisense retrotransposon fragments (Aravin et al. 2007b; Brennecke et al. 2007). In the presence of active transposons, sense piRNAs are also found, which are thought to be derived from the transposon, and may help to amplify the more abundant antisense piRNAs (Gunawardane et al. 2007). It is generally assumed that transposons have evolved to infect the germ line, which is where piRNAs mount a host defense (Sarot et al. 2004; Brennecke et al. 2007).

On the basis of our results in plants, however, it is possible that not only are piRNA expressed in the germ line, but so are the transposons they target, deliberately released by the host to provide a small RNA signal and efficiently silence themselves. In Drosophila, somatic follicular cells adjacent to female germ-line stem cells are the site of long terminal repeat (LTR) retrotransposon Gypsy expression (Sarot et al. 2004; Brennecke et al. 2007). Furthermore, piRNA-mediated repression of hybrid dysgenesis by non-LTR retrotransposons depends on expression of full-length autonomous elements in nurse cell nuclei adjacent to the oocyte (Chambeyron et al. 2008). It is possible that piRNA or TE primary transcripts are generated in these companion cells and then translocated into the oocyte.

In mammals, deep sequencing of small RNA from oocytes has revealed abundant 25-29-nucleotide piRNA and similarly abundant 21-nucleotide endogenous siRNA (Tam et al. 2008; Watanabe et al. 2008). In these reports, the vast majority of 21-nucleotide siRNAs were from retrotransposons, as we have observed in plant sperm cells (Slotkin et al. 2009). It is not yet known at which stage of germ-line development these siRNAs arise; however, the transient loss of heterochromatin in germ cells around E11.5 (Hajkova et al. 2008) could provide the window of opportunity for retrotransposon transcripts and associated 21-nucleotide siRNA to be produced.

\section{REPROGRAMMING AND CANCER}

Reprogramming is not limited to the germ cells. Indeed, similarities in expression profiles among germ cells, stem cells, and cancer cells have, in part, led to the "cancer stem cell" hypothesis (Reya et al. 2001), and TEs are significantly up-regulated in these contexts. Cultured cells in general, and especially cancer cells, lose methylation genome-wide, largely from transposons and repeats. In addition, developmental reprogramming itself, in which a handful of transcription factors induce a pluripotent state, is greatly enhanced by inhibiting DNA methylation genome-wide (Meissner et al. 2008; Mikkelsen et al. 2008). In the developing embryo immediately after fertilization, DNA methylation is lost (passively) from the female pronucleus and (actively) from the male pronucleus, only being regained after differentiation of the trophoblast, which remains hypomethylated (Branco et al. 2008). In both the developing oocyte and the developing embryo, these cycles of demethylation and remethylation 
depend on down-regulation and mislocalization of the DNA methyltransferases Dnmt1, Dnmt3a, and Dnmt3b, resembling reprogramming in pollen in this respect.

The activation of transposable elements is thus programmed and "intentional" in somatic and germ cells in both plants and animals. Rather than representing a victory of parasite over host, we propose that the transposons are activated transiently at a time when they need to be silenced and that silencing occurs efficiently via RNAi. Importantly, when the RNAi machinery is inactivated in piwi mutants in animals or in certain argonaute mutants in plants (Nonomura et al. 2007), sterility ensues presumably because of the very significant transposon load. Although certainly speculative, it is hard to ignore the potential for selectively targeting cancer cells by loss of transposon RNAi.

\section{CONCLUSIONS}

The genetics and genomics of heterochromatin in fission yeast and plants have expanded our understanding of the establishment, maintenance, and inheritance of epigenetic marks that render heterochromatin transcriptionally silent. Heterochromatin inheritance in fission yeast is tightly coupled to the cell cycle via the phosphomethyl switch, which releases transcriptional repression from heterochromatic repeats. Their transcripts are then rapidly turned over during $\mathrm{S}$ phase by RNAi, ensuring that newly synthesized chromatin is once again fully decorated with heterochromatic marks and is returned to transcriptional silencing.

In long-term plant cell culture, similarly to immortalized animal cell lines and cancer cell lines, there is a substantial, yet very localized, loss of heterochromatic silencing specific to families of TEs, in parallel with hypermethylation of gene coding and regulatory regions. The latter represents epigenetic restructuring of the genome in immortalized cells and can lead to mitotically heritable changes in gene expression programs. A novel class of 21-nucleotide siRNA corresponding to the epigenetically reactivated repeats appears in plant cell culture as well as in mutants in metl (Dnmt1) and $d d m 1$ (Lshl) and in wild-type pollen.

Down-regulation of chromatin regulators in the pollen $\mathrm{VN}$ represents a form of epigenetic reprogramming that leads to reactivation of TEs in companion cells that support the germ cells without contribution to the next generation, revealing the TE content of the parental genome and silencing them in the sperm via mobile siRNA. This likely ensures proper heterochromatic silencing following fertilization.

\section{ACKNOWLEDGMENTS}

We thank M.W. Vaughn and W.R. McCombie for their help with high-throughput sequencing and analysis. The work described here is funded by grants from the National Institutes of Health (GM076396) and the National Science Foundation project (DBI-042165) to R.A.M. and a National Institutes of Health postdoctoral fellowship (F32 CA125977) to R.K.S.

\section{REFERENCES}

Aravin, A.A., Hannon, G.J., and Brennecke, J. 2007a. The PiwipiRNA pathway provides an adaptive defense in the transposon arms race. Science 318: 761-764.

Aravin, A.A., Sachidanandam, R., Girard, A., Fejes-Toth, K., and Hannon, G.J. 2007b. Developmentally regulated piRNA clusters implicate MILI in transposon control. Science 316: 744-747.

Becker, J.D., Boavida, L.C., Carneiro, J., Haury, M., and Feijo, J.A. 2003. Transcriptional profiling of Arabidopsis tissues reveals the unique characteristics of the pollen transcriptome. Plant Physiol. 133: 713-725.

Borges, F., Gomes, G., Gardner, R., Moreno, N., McCormick, S., Feijo, J.A., and Becker, J.D. 2008. Comparative transcriptomics of Arabidopsis thaliana sperm cells. Plant Physiol. 148: $1168-1181$.

Branco, M.R., Oda, M., and Reik, W. 2008. Safeguarding parental identity: Dnmt1 maintains imprints during epigenetic reprogramming in early embryogenesis. Genes Dev. 22: $1567-1571$.

Brennecke, J., Aravin, A.A., Stark, A., Dus, M., Kellis, M., Sachidanandam, R., and Hannon, G.J. 2007. Discrete small RNA-generating loci as master regulators of transposon activity in Drosophila. Cell 128: 1089-1103.

Brodersen, P. and Voinnet, O. 2006. The diversity of RNA silencing pathways in plants. Trends Genet. 22: 268-280.

Chambeyron, S., Popkova, A., Payen-Groschene, G., Brun, C., Laouini, D., Pelisson, A., and Bucheton, A. 2008. piRNAmediated nuclear accumulation of retrotransposon transcripts in the Drosophila female germline. Proc. Natl. Acad. Sci. 105: 14964-14969.

Chen, E.S., Zhang, K., Nicolas, E., Cam, H.P., Zofall, M., and Grewal, S.I.S. 2008. Cell cycle control of centromeric repeat transcription and heterochromatin assembly. Nature 451: 734 737.

Colmenares, S.U., Buker, S.M., Buhler, M., Dlakic, M., and Moazed, D. 2007. Coupling of double-stranded RNA synthesis and siRNA generation in fission yeast RNAi. Mol. Cell 27: 449-461.

Engel, M.L., Holmes-Davis, R., and McCormick, S. 2005. Green sperm. Identification of male gamete promoters in Arabidopsis. Plant Physiol. 138: 2124-2133.

Fischle, W., Wang, Y.M., and Allis, C.D. 2003. Binary switches and modification cassettes in histone biology and beyond. Nature 425: 475-479.

Grandbastien, M.A., Spielmann, A., and Caboche, M. 1989. Tnt1, a mobile retroviral-like transposable element of tobacco isolated by plant-cell genetics. Nature 337: 376-380.

Gunawardane, L.S., Saito, K., Nishida, K.M., Miyoshi, K., Kawamura, Y., Nagami, T., Siomi, H., and Siomi, M.C. 2007. A slicer-mediated mechanism for repeat-associated siRNA 5' end formation in Drosophila. Science 315: 1587-1590.

Hajkova, P., Ancelin, K., Waldmann, T., Lacoste, N., Lange, U.C., Cesari, F., Lee, C., Almouzni, G., Schneider, R., and Surani, M.A. 2008. Chromatin dynamics during epigenetic reprogramming in the mouse germ line. Nature 452: 877881.

Heitz, E. 1928. Das heterochromatin der Moose. Jahrb. Wiss. Botanik. 69: 762-818.

Henderson, I.R. and Jacobsen, S.E. 2007. Epigenetic inheritance in plants. Nature 447: 418-424.

Hirochika, H. 1993. Activation of tobacco retrotransposons during tissue-culture. EMBO J. 12: 2521-2528.

Holm, T.M., Jackson-Grusby, L., Brambrink, T., Yamada, Y., Rideout III, W.M., and Jaenisch, R. 2005. Global loss of imprinting leads to widespread tumorigenesis in adult mice. Cancer Cell 8: 275-285.

Honys, D. and Twell, D. 2004. Transcriptome analysis of haploid male gametophyte development in Arabidopsis. Genome Biol. 5: R85.

Horn, P.J., Bastie, J.N., and Peterson, C.L. 2005. A Rik1-associated, cullin-dependent E3 ubiquitin ligase is essential for heterochromatin formation. Gene Dev 19: 1705-1714. 
Irvine, D.V., Zaratiegui, M., Tolia, N.H., Goto, D.B., Chitwood, D.H., Vaughn, M.W., Joshua-Tor, L., and Martienssen, R.A. 2006. Argonaute slicing is required for heterochromatic silencing and spreading. Science 313: 1134-1137.

Jacobsen, S.E., Sakai, H., Finnegan, E.J., Cao, X., and Meyerowitz, E.M. 2000. Ectopic hypermethylation of flower-specific genes in Arabidopsis. Curr. Biol. 10: 179-186.

Kaeppler, S.M., Kaeppler, H.F., and Rhee, Y. 2000. Epigenetic aspects of somaclonal variation in plants. Plant Mol. Biol. 43: $179-188$.

Kim, S.M. and Huberman, J.A. 2001. Regulation of replication timing in fission yeast. EMBO J. 20: 6115-6126.

Kinoshita, T., Miura, A., Choi, Y., Kinoshita, Y., Cao, X., Jacobsen, S.E., Fischer, R.L., and Kakutani, T. 2004. Oneway control of FWA imprinting in Arabidopsis endosperm by DNA methylation. Science 303: 521-523.

Kloc, A. and Martienssen, R. 2008. RNAi, heterochromatin and the cell cycle. Trends Genet. 24: 511-517.

Kloc, A., Zaratiegui, M., Nora, E., and Martienssen, R. 2008 RNA interference guides histone modification during the $\mathrm{S}$ phase of chromosomal replication. Curr. Biol. 18: 490-495.

Lippman, Z. and Martienssen, R. 2004. The role of RNA interference in heterochromatic silencing. Nature 431: 364-370.

Lippman, Z., May, B., Yordan, C., Singer, T., and Martienssen, R. 2003. Distinct mechanisms determine transposon inheritance and methylation via small interfering RNA and histone modification. PLoS Biol. 1: E67.

Lippman, Z., Gendrel, A.V., Black, M., Vaughn, M.W., Dedhia, N., McCombie, W.R., Lavine, K., Mittal, V., May, B., Kasschau, K.D., et al. 2004. Role of transposable elements in heterochromatin and epigenetic control. Nature 430: 471-476.

Martienssen, R.A. and Colot, V. 2001. DNA methylation and epigenetic inheritance in plants and filamentous fungi. Science 293: 1070-1074.

Meissner, A., Mikkelsen, T.S., Gu, H.C., Wernig, M., Hanna, J., Sivachenko, A., Zhang, X.L., Bernstein, B.E., Nusbaum, C., Jaffe, D.B., et al. 2008. Genome-scale DNA methylation maps of pluripotent and differentiated cells. Nature 454: 766-770.

Mikkelsen, T.S., Hanna, J., Zhang, X.L., Ku, M.C., Wernig, M., Schorderet, P., Bernstein, B.E., Jaenisch, R., Lander, E.S., and Meissner, A. 2008. Dissecting direct reprogramming through integrative genomic analysis. Nature 454: 49-55.

Nonaka, N., Kitajima, T., Yokobayashi, S., Xiao, G.P., Yamamoto, M., Grewal, S.I.S., and Watanabe, Y. 2002. Recruitment of cohesin to heterochromatic regions by Swi6/HP1 in fission yeast. Nat. Cell Biol. 4: 89-93.

Nonomura, K., Morohoshi, A., Nakano, M., Eiguchi, M., Miyao, A., Hirochika, H., and Kurata, N. 2007. A germ cell specific gene of the ARGONAUTE family is essential for the progression of premeiotic mitosis and meiosis during sporogenesis in rice. Plant Cell 19: 2583-2594

Pina, C., Pinto, F., Feijo, J.A., and Becker, J.D. 2005. Gene family analysis of the Arabidopsis pollen transcriptome reveals biological implications for cell growth, division control, and gene expression regulation. Plant Physiol. 138: 744-756.

Reya, T., Morrison, S.J., Clarke, M.F., and Weissman, I.L. 2001. Stem cells, cancer, and cancer stem cells. Nature 414: 105-111.

Sarot, E., Payen-Groschene, G., Bucheton, A., and Pelisson, A.
2004. Evidence for a piwi-dependent RNA silencing of the gypsy endogenous retrovirus by the Drosophila melanogaster flamenco gene. Genetics 166: 1313-1321.

Schmid, M., Davison, T.S., Henz, S.R., Pape, U.J., Demar, M., Vingron, M., Scholkopf, B., Weigel, D., and Lohmann, J.U. 2005. A gene expression map of Arabidopsis thaliana development. Nat. Genet. 37: 501-506.

Schwab, R., Ossowski, S., Riester, M., Warthmann, N., and Weigel, D. 2006. Highly specific gene silencing by artificial microRNAs in Arabidopsis. Plant Cell 18: 1121-1133.

Singer, T., Yordan, C., and Martienssen, R.A. 2001. Robertson's Mutator transposons in A. thaliana are regulated by the chromatin-remodeling gene decrease in DNA methylation (DDM1). Genes Dev. 15: 591-602.

Slotkin, R.K., Vaughn, M., Borges, F., Tanurdžić M., Becker, J.D., Feijó, J.A., and Martienssen, R.A. 2009. Epigenetic reprogramming and small RNA silencing of transposable elements in pollen. Cell 136: 461-472.

Smith, P.A. and Corces, V.G. 1995. The suppressor of hairywing protein regulates the tissue-specific expression of the Drosophila gypsy retrotransposon. Genetics 139: 215-228.

Sundaresan, V., Springer, P., Volpe, T., Haward, S., Jones, J.D., Dean, C., Ma, H., and Martienssen, R. 1995. Patterns of gene action in plant development revealed by enhancer trap and gene trap transposable elements. Genes Dev. 9: 1797-1810.

Tam, O.H., Aravin, A.A., Stein, P., Girard, A., Murchison, E.P., Cheloufi, S., Hodges, E., Anger, M., Sachidanandam, R., Schultz, R.M., and Hannon, G.J. 2008. Pseudogene-derived small interfering RNAs regulate gene expression in mouse oocytes. Nature 453: 534-538.

Tanurdžić, M., Vaughn, M.W., Jiang, H., Lee, T.-J., Slotkin, R.K., Sosinski, B., Thompson, W.F., Doerge, R.W., and Martienssen, R.A. 2008. Epigenomic consequences of immortalized plant cell suspension culture. PLoS Biol. 6: e302.

Vaughn, M.W., Tanurdžić, M., Lippman, Z., Jiang, H., Carrasquillo, R., Rabinowicz, P.D., Dedhia, N., McCombie, W.R., Agier, N., Bulski, A., et al. 2007. Epigenetic natural variation in Arabidopsis thaliana. PLoS Biol. 5: e174.

Verdel, A., Jia, S.T., Gerber, S., Sugiyama, T., Gygi, S., Grewal, S.I.S., and Moazed, D. 2004. RNAi-mediated targeting of heterochromatin by the RITS complex. Science 303: 672-676.

Volpe, T., Schramke, V., Hamilton, G.L., White, S.A., Teng, G., Martienssen, R.A., and Allshire, R.C. 2003. RNA interference is required for normal centromere function in fission yeast. Chromosome Res. 11: 137-146.

Volpe, T.A., Kidner, C., Hall, I.M., Teng, G., Grewal, S.I.S., and Martienssen, R.A. 2002. Regulation of heterochromatic silencing and histone H3 lysine-9 methylation by RNAi. Science 297: 1833-1837.

Watanabe, T., Totoki, Y., Toyoda, A., Kaneda, M., KuramochiMiyagawa, S., Obata, Y., Chiba, H., Kohara, Y., Kono, T., Nakano, T., et al. 2008. Endogenous siRNAs from naturally formed dsRNAs regulate transcripts in mouse oocytes. Nature 453: 539-543.

Zaratiegui, M., Irvine, D.V., and Martienssen, R.A. 2007. Noncoding RNAs and gene silencing. Cell 128: 763-776.

Zhu, J.H., Kapoor, A., Sridhar, V.V., Agius, F., and Zhu, J.K. 2007. The DNA glycosylase/lyase ROS1 functions in pruning DNA methylation patterns in Arabidopsis. Curr. Biol. 17: 54-59. 


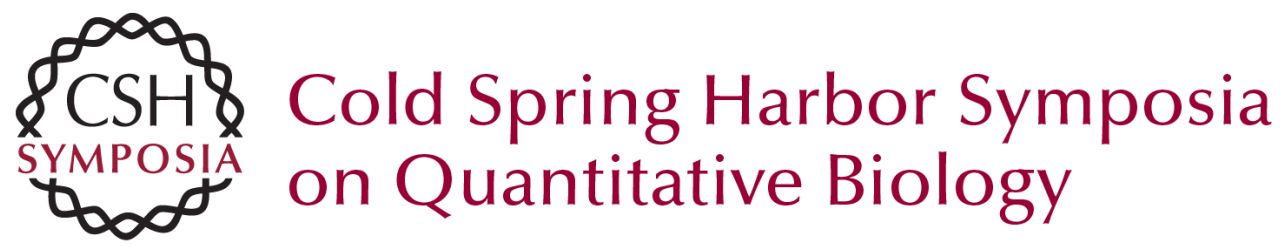

\section{Epigenetic Inheritance and Reprogramming in Plants and Fission Yeast}

R.A. Martienssen, A. Kloc, R.K. Slotkin, et al.

Cold Spring Harb Symp Quant Biol 2008 73: 265-271 originally published online March 27, 2009

Access the most recent version at doi:10.1101/sqb.2008.73.062

References This article cites 54 articles, 22 of which can be accessed free at:

http://symposium.cshlp.org/content/73/265.full.html\#ref-list-1

\section{License}

Email Alerting Receive free email alerts when new articles cite this article - sign up in the box at the Service top right corner of the article or click here. 